\title{
JACOBIANS FOR MEASURES IN COSET SPACES
}

\author{
by $\mathrm{S}$. ŚWIERCZKOWSKI
}

(Received 10 December, 1959)

Let $G$ be a locally compact topological group, let $H$ be a closed subgroup and let $G / H$ be the space of left cosets $\bar{x}=x H$ with the natural topology. We denote by $\mu$ a non-negative measure in $G / H$ defined on the ring of Baire sets. $G$ acts by left multiplication as a transitive group of homeomorphisms on $G / H$ : Every $t \in G$ defines the homeomorphism $\bar{x} \rightarrow t \bar{x}=\overline{t x}$. We write, for $E \subset G / H, t E=\{t \bar{x}: \bar{x} \in E\}$. The measure $\mu$ is called stable (cf. [3], [4]) if from $t \in G, E \subset G / H$ and $\mu(E)=0$ follows $\mu(t E)=0$. We say that $\mu$ is locally finite [3], [5] if every set of positive measure contains a subset of positive finite measure.

Let us write $\xi$ for a typical element of $H$ and let $d \xi$ indicate integration with respect to a left invariant Haar measure in $H$. Evidently, if $f(x)$ is a non-negative Baire function on $G$, then $\int f(x \xi) d \xi$ is everywhere defined and, considered as a function of $x$, constant on cosets $x H$; thus it is really a function of $\vec{x}$ (see [2]). We write

$$
\bar{f}(\bar{x})=\int \bar{f}(x \xi) d \xi
$$

Then $\bar{f}(\bar{x})$ is a non-negative Baire function on $G / H$ [2], [3].

We call $\mu$ inherited if there is a measure $\tilde{\mu}$ in $G$ defined on Baire sets which is absolutely continuous with respect to the Haar measure and such that, for every non-negative Baire function $f(x)$ on $G$,

$$
\int f(x) d \tilde{\mu}(x)=\int f(\bar{x}) d \mu(\bar{x})
$$

Such measures always do exist (cf. [2]). In particular, every stable measure $\mu$ which is finite on some open set is inherited [3], [4].

Given a topological space $X$, we shall denote by $B_{+}(X)$ the class of all non-negative extended real-valued Baire functions on $X$ (extended real numbers include $\pm \infty$ ). $L B_{+}(X)$ will stand for the class of all functions $f(x)$ such that $f(x) g(x) \in B_{+}(X)$ whenever $g(x) \in B_{+}(X)$ (locally Baire functions). A set $Q \subset X$ will be called an $L B$-set if there is a function $f(x) \in L B_{+}(x)$ with $f(x)=0$ or 1 and $f(x)=1$ if and only if $x \in Q$. Given a measure $\mu$ on Baire subsets of $X$, we generalize the notion of a set of measure zero to $L B$-sets as follows : $\mu(Q)=0$ if and only if $\mu(Q \cap E)=0$ for every Baire set $E$. (We note that $Q \cap E$ is a Baire set.) In this generalized sense we use the phrase "almost everywhere with respect to $\mu$ ", which will be denoted also by $[\mu]$.

A function $J(t, \bar{x}) \in L B_{+}(G \times G / H)$ is called a Jacobian for $\mu$ (or $\mu$-Jacobian) if, for every $f(\bar{x}) \in B_{+}(G / H)$ and $t \in G$,

$$
\int f\left(t^{-1} \bar{x}\right) d \mu(\bar{x})=\int f(\bar{x}) J(t, \bar{x}) d \mu(\bar{x})
$$


It is not difficult to check (see [2], [4]) that, for each $s, t \in G$, a $\mu$-Jacobian satisfies

$$
J(s t, \bar{x})=J(s, t \bar{x}) J(t, \bar{x})
$$

for almost all $\bar{x}$ with respect to the measure $\mu$. A Jacobian is called exact if the above equality holds identically for every $\bar{x}$. It is known that, for a stable locally finite inherited measure $\mu$, there always exists an exact positive and finite-valued Jacobian (see [3], and Lemma 3 below). The class of exact Jacobians was characterized in [3] by functional equations. We give in this note an analogous characterization of the class of all Jacobians for these measures.

We denote by $m$ a left-invariant Haar measure in $G$. The phrases " almost all ", " almost every" etc. will be used, unless stated otherwise, with respect to the Haar measures $m$, $m^{2}=m \times m$ and $m^{3}=m \times m \times m$ in $G, G^{2}=G \times G$ and $G^{3}=G \times G \times G$ respectively. These phrases will occasionally be replaced by the symbols $[m],\left[m^{2}\right]$ and $\left[m^{3}\right]$. For convenience we shall sometimes write $J(t, x)$ instead of $J(t, \bar{x})$, assuming always that $J(t, x)=J(t, x \xi)$ for all $\xi \in H$. Let $\Delta(x)$ and $\delta(\xi)$ denote the modular functions for $m$ and for the left invariant Haar measure in $H$.

Theorem. A function $J(t, \bar{x}) \in L B_{+}(G \times G / H)$ is a Jacobian for some stable locally finite inherited measure in $G / H$ if and only if it satisfies the following conditions :

$(\alpha) 0<J(t, x)<\infty$ for almost all pairs $\langle t, x\rangle \in G^{2}$,

( $\beta$ ) for every $s, t \in G, J(s t, x)=J(s, t x) J(t, x)$ holds for almost all $x \in G$,

$(\gamma)$ for every $\xi \in H, J\left(t \xi x^{-1}, x\right) \Delta(\xi)=J\left(t x^{-1}, x\right) \delta(\xi)$ holds for almost all pairs $\langle t, x\rangle \in G^{2}$.

In the proof we shall use some earlier results of A. M. Macbeath and the present author. We list them here in Lemmas 1-4.

Lemma 1. A measure $\tilde{\mu}$ is associated by (1) with a stable locally finite inherited measure $\mu$ if and only if the Radon-Nikodym derivative $[1, \S 32, \mathrm{p} .133] d \tilde{\mu} / d m=h(x) \in L B_{+}(G)$ is such that

(i) $0<h(x)<\infty \quad[m]$,

(ii) for every $\xi \in H, h(x \xi) \Delta(\xi)=h(x) \delta(\xi)$ holds for almost every $x \in G$.

The function $h(x)$ is called a $\mu$-factor function [2], [3].

Lemma 2. For every stable locally finite inherited measure $\mu$ there is a $\mu$-factor function $h_{0}(x)$ such that both (i) and (ii) in Lemma 1 hold identically for all $x \in G, \xi \in H$ (see [3]).

LEMMA 3. Given a $\mu$-factor function $h_{0}(x)$ satisfying the condition of Lemma 2 , the formula

$$
J_{0}(t, x)=h_{0}(t x) / h_{0}(x)
$$

defines an exact $\mu$-Jacobian. We note that, for each $t, h_{0}(t x) / h_{0}(x)$ is constant on the coset $x H$ since (ii) holds identically also for $t x$ in place of $x$ and for all $\xi \in H$ (see [3]).

LEMma 4. The mapping $f \rightarrow \bar{f}$ maps $B_{+}(G)$ onto $B_{+}(G / H)$ (see [2], [3]).

We denote by $\phi$ the canonical mapping of $G$ onto $G / H,(\phi(x)=\bar{x})$. Then we obtain from Lemmas 1 and 4 the

Corollary. For any LB-set $Q \subset G / H, \mu(Q)=0$ is equivalent to $m\left(\phi^{-1}(Q)\right)=0$.

To see this we note that $m\left(\phi^{-1}(Q)\right)=0$ means, by condition (i) of Lemma 1 , that the $\tilde{\mu}$-integral of every $f \in B_{+}\left(\phi^{-1}(Q)\right)$ is equal to zero. Similarly, $\mu(Q)=0$ means that the $\mu$ integral of every $g \in B_{+}(Q)$ is equal to zero. Thus, by formula (1) and Lemma 4 , both conditions mean the same. 
LEMMA 5. Each of the transformations $S\langle t, x\rangle=\langle t x, x\rangle, T_{v}\langle t, x\rangle=\langle t v, x\rangle, \quad(v \in G$ fixed), $R_{1}\langle s, t, x\rangle=\langle s t x, t x, x\rangle, R_{2}\langle s, t, x\rangle=\langle s, t x, x\rangle$ of $G^{2}$ or $G^{3}$ onto itself maps sets of measure zero on sets of measure zero.

Proof. Since the right and left Haar measures are absolutely continuous one with respect to the other [1], we may assume in this proof that the considered measures are right invariant. Then the transformations are all measure-preserving. This is well known for $S[1, \S 59$, Theorem B] and it is trivial for $T_{v}$. To prove that the $R_{i}$ are also measure preserving, we note that, if we have two spaces $U, W$ with measures $m_{1}$ and $m_{2}$ and $S(u)$ is a measure preserving transformation of $U$ into itself, then the transformation $\langle u, w\rangle \rightarrow\langle S(u), w\rangle$ preserves the measure $m_{1} \times m_{2}$ in $U \times W$ (because every rectangle is transformed into a rectangle of the same measure). Now each $R_{i}$ can be obtained by combining transformations of the above kind where $S$ is the transformation $S\langle t, x\rangle=\langle t x, x\rangle$. This proves the lemma.

We shall frequently apply a consequence of Fubini's theorem stating that a subset of a product space has measure zero if and only if every section has measure zero $[1, \S 36$, Theorem A].

Necessity of $(\alpha),(\beta)$ and $(\gamma)$. Suppose that $J(t, \bar{x}) \in L B_{+}(G \times G / H)$ is a $\mu$-Jacobian. By Lemma 3 , there is an everywhere finite and positive exact $\mu$-Jacobian $J_{0}(t, \bar{x})$. For every $t, J_{0}(t, \bar{x})=J(t, \bar{x})[\mu]$, by (2), and hence $J_{0}(t, x)=J(t, x)$ for almost all $x$, by the corollary to Lemma 4. Thus, by Fubini's theorem, we have $(\alpha)$.

Condition $(\beta)$ is an immediate consequence of $(3)$ and of the same corollary. To prove ( $\gamma$ ) observe that, for the $\mu$-Jacobian $J_{0}(t, x),(\gamma)$ follows from condition (ii) of Lemma 1 . Now, for each $t, J_{0}(t, x)=J(t, x)$ for almost all $x$; hence $J_{0}(t, x)=J(t, x)\left[m^{2}\right]$. Using the transformations $S$ and $T_{v}$ of Lemma 5, we have, for each $v \in G, J_{0}\left(t v^{-1} x^{-1}, x\right)=J\left(t v^{-1} x^{-1}, x\right)$ for almost all pairs $\langle t, x\rangle$. Putting $v=\xi^{-1}$ and $e$ in turn we derive $(\gamma)$ from the analogous equality for $J_{0}(t, x)$.

Sufficiency of $(\alpha),(\beta)$ and $(\gamma)$. We assume $(\alpha),(\beta)$ and $(\gamma)$, and we shall prove that $J(t, \bar{x}) \in L B_{+}(G \times G / H)$ is a Jacobian for a certain inherited stable locally finite measure $\mu$.

Lemma 6. There is a function $h(s) \in L B_{+}(G)$ such that, for every $\sigma$-compact open subgroup $\Gamma \subset G$,

(a) $0<h(s)<\infty$ for almost all $s \in \Gamma$,

(b) for almost all $t \in \Gamma$, there is a number $0<c_{t}<\infty$ such that

$$
h(s)=c_{t} J\left(s t^{-1}, t\right) \text { for almost all } s \in \Gamma .
$$

Denote by $\Phi(\Gamma)$ the class of Baire functions which are defined on $\Gamma$ and have the properties $(a)$ and $(b)$. We establish first some properties of this class. (In the proofs of A and B below we assume that all transformations and functions are restricted to $\Gamma$.)

A. The class $\Phi(\Gamma)$ is not empty.

Using the transformation $S\langle t, x\rangle=\langle t x, x\rangle$ we derive from $(\alpha)$, by Lemma 5 , that $0<J\left(t x^{-1}, x\right)<\infty\left[m^{2}\right]$. Hence the set $M_{0}$ of all $x \in \Gamma$ such that $0<J\left(t x^{-1}, x\right)<\infty$ holds for almost all $t$, satisfies $m\left(\Gamma-M_{0}\right)=0$.

Applying Fubini's theorem and the transformation $R_{1}\langle s, t, x\rangle=\langle s t x, t x, x\rangle$, we obtain from $(\beta)$ that

$$
J\left(s x^{-1}, x\right)=J\left(s t^{-1}, t\right) J\left(t x^{-1}, x\right) \quad\left[m^{3}\right]
$$


Hence, if $M_{1}$ denotes the set of all $x \in \Gamma$, such that the above equality holds for almost all $\langle s, t\rangle \in \Gamma \times \Gamma$, then $m\left(\Gamma-M_{1}\right)=0$. Thus $M_{0} \cap M_{1} \neq 0$. Let $x_{0} \in M_{0} \cap M_{1}$. Then, for almost all $t \in \Gamma, 0<J\left(t x_{0}^{-1}, x_{0}\right)<\infty$ and

$$
J\left(s x_{0}^{-1}, x_{0}\right)=J\left(s t^{-1}, t\right) J\left(t x_{0}^{-1}, x_{0}\right) \text { for almost all } s \in \Gamma .
$$

Define $c_{t}=J\left(t x_{0}^{-1}, x_{0}\right), h(s)=J\left(s x_{0}^{-1}, x_{0}\right)$. Then $0<c_{t}<\infty[m]$ and $0<h(s)<\infty[m]$, and (4) holds by (5). This proves $A$. $s \in \Gamma$.

B. If $g, h \in \Phi(\Gamma)$, then there is exactly one constant $c$ such that $g(s)=h(s) \cdot c$ for almost all

Suppose that $\left\{c_{t}^{0}\right\}$ is the set of numbers which appear in condition $(b)$ for $g$ in place of $h$. There is a $t \in \Gamma$, such that (4) holds for both $g$ and $h$, and then, by $(a), g(s)=h(s) \cdot c$, where $c=c_{t}^{0} / c_{t}$. The uniqueness of $c$ follows from condition $(a)$.

We observe that $\Gamma_{0} \subset \Gamma_{1}$ implies $\Phi\left(\Gamma_{1}\right) \subset \Phi\left(\Gamma_{0}\right)$. Hence from B we have

C. If $\Gamma_{0} \subset \Gamma_{1}$ are $\sigma$-compact open subgroups of $G$ and $h_{i}(s) \in \Phi\left(\Gamma_{i}\right),(i=0,1)$, then there is a unique constant $c_{0}$ such that $h_{1}(s) \cdot c_{0}=h_{0}(s)[m]$ on $\Gamma_{0}$. Obviously $h_{1}(s) \cdot c_{0}$ belongs to $\Phi\left(\Gamma_{1}\right)$.

Proof of Lemma 6. Let $\Gamma_{0}$ be a fixed $\sigma$-compact open subgroup of $G$ and let $h_{0}{ }^{*} \in \Phi\left(\Gamma_{0}\right)$. For every $\sigma$-compact group $\Gamma \subset G$ which contains $\Gamma_{0}$ we denote by $h^{*}$ a function in $\Phi(\Gamma)$ which satisfies $h^{*}(s)=h_{0}{ }^{*}(s)[m]$ on $\Gamma_{0}$. Such a function exists by C. If we have $\Gamma_{i}$ in place of $\Gamma$, the corresponding function $h^{*}$ will be denoted by $h_{i}^{*}$. We now define $h(s)$ on $G$, defining it on each $\Gamma_{0}$-coset. First we put $h(s)=h_{0}{ }^{*}(s)$ on $\Gamma_{0}$. For each coset $x_{i} \Gamma_{0}$, denote by $\Gamma_{i}$ the group generated by the set $\Gamma_{0} \cup x_{i} \Gamma_{0}$. This is a $\sigma$-compact group and we define $h(s)=h_{i}^{*}(s)$ on $x_{i} \Gamma_{0}$.

It remains to verify that conditions $(a)$ and $(b)$ hold. Obviously we may assume that $\Gamma$ contains $\Gamma_{0}$, so that it is a union of $\Gamma_{0}$-cosets. We shall prove that, for every coset $x_{i} \Gamma_{0} \subset \Gamma$ $h(s)=h^{*}(s)[m]$ on $x_{i} \Gamma_{0}$. Since $\Gamma$ is $\sigma$-compact, it contains at most a countable number of $\Gamma_{0}$-cosets; hence $h(s)=h^{*}(s)[m]$ on $\Gamma$. This will prove the lemma, because $h^{*}$ satisfies $(a)$ and $(b)$ by definition. Since $\Gamma_{i}$ is a subgroup of $\Gamma$, there is, by $\mathrm{C}$, a unique constant $c_{i}$ such that $h^{*}(s) \cdot c_{i}=h_{i}^{*}(s)[m]$ on $\Gamma_{i}$. We have, on $\Gamma_{0}, h^{*}(s) \cdot c_{i}=h_{i}^{*}(s)=h_{0}^{*}(s)=h^{*}(s)[m]$. Thus $c_{i}=1$ and $h^{*}(s)=h_{i}^{*}(s)[m]$ on $\Gamma_{i}$. In particular this is true on $x_{i} \Gamma_{0}$, where $h_{i}^{*}(s)=h(s)$. This proves the lemma.

We show that the function $h(s)$ constructed above satisfies condition (ii) of Lemma 1 . Obviously it is enough to verify that (ii) holds on every $\sigma$-compact open subgroup $\Gamma \subset G$. Let $v \in \Gamma$. We have, from Lemma 6, for almost all $t \in \Gamma$,

$$
h(s)=c_{t} J\left(s t^{-1}, t\right) \text { for almost all } s \in \Gamma \text {; }
$$

hence $h(s v)=c_{t} J\left(s v t^{-1}, t\right)$ for almost all $s \in \Gamma$. Consequently

$$
h(s v) J\left(s t^{-1}, t\right)=h(s) J\left(s v t^{-1}, t\right) \text { for almost all pairs }\langle s, t\rangle,
$$

and if we take $v=\xi \in H$, then the above condition and $(\gamma)$ imply (ii).

Since $h$ is almost everywhere finite and positive, it defines, by Lemma 1 , a stable locally finite inherited measure $\mu$ on $G / H$. It remains to show that $J(t x)$ is a $\mu$-Jacobian. This will follow if we prove 
LEMma 7. On every open o-compact group $\Gamma \subset G$ we have, for all $s \in \Gamma, J(s, v)=J_{0}(s, v)$ for almost all $v \in \Gamma$, where $J_{0}(s, v)$ is the $\mu$-Jacobian given by Lemma 3 .

Proof. (We restrict all functions and transformations to $\Gamma$.) Condition $(\beta)$ implies that $J(s v, t)=J(s, v t) J(v, t)\left[m^{3}\right]$. Applying the transformation $R_{2}\langle s, v, t\rangle=\langle s, v t, t\rangle$, we have, by Lemma $5, J\left(s v t^{-1}, t\right)=J(s, v) J\left(v t^{-1}, t\right)\left[m^{3}\right]$. Thus, by property $(b)$ of $h(s)$,

$$
h(s v)=J(s, v) h(v) \quad\left[m^{2}\right] .
$$

Now, if $h_{0}$ is a $\mu$-factor function which satisfies the condition of Lemma 2 , then $h_{0}(s)=h(s)[m]$ (because each of these functions is a Radon-Nikodym derivative $\tilde{d \mu} / d m$ ). Consequently $h_{0}(s v)=J(s, v) h_{0}(v)\left[m^{2}\right]$. This means that, for almost all $s \in \Gamma, J(s, v)=J_{0}(s, v)$ holds for almost all $v \in \Gamma$, where $J_{0}$ is the exact $\mu$-Jacobian given by Lemma 3. If $M \subset \Gamma$ is the set of all $s$ such that $J(s, v)=J_{0}(s, v)$ for almost all $v \in \Gamma$, then $m(\Gamma-M)=0$ and we have to show that $M=\Gamma$. Now, if $s, t \in M$, then, by $(\beta)$ and since $J_{0}$ is an exact Jacobian, we have $s t \in M$. This proves that $M=\Gamma$, because every element $x \in \Gamma$ can be represented in the form $x=s t$, where $s, t \in M$; it is enough to take $s \in M \cap x M^{-1}$.

\section{REFERENCES}

1. P. R. Halmos, Measure theory, New York 1951.

2. A. M. Macbeath and S. Swierczkowski, Measures in homogeneous spaces, Fundamenta Math., 49 (1960), 15-24.

3. A. M. Macbeath and S. Swierczkowski, Inherited measures ; to appear in Proc. Roy. Soc. Edinburgh.

4. S. Swierczkowski, Measures equivalent to the Haar measure, Proc. Glasgow Math. Assoc., 4 (1960), 157-162.

5. A. C. Zaanen, A note on measure theory, Nieuw Arch. Wisk. (3) 6 (1958), 58-65.

\section{The University}

Glasgow 